\title{
Impact of age on extracorporeal membrane oxygenation survival of patients with cardiac failure
}

\author{
This article was published in the following Dove Press journal: \\ Clinical Interventions in Aging \\ 24 August 2017 \\ Number of times this article has been viewed
}

\section{Su Nam Lee' \\ Min Seop Jo² \\ Ki-Dong Yoo'}

'Department of Internal Medicine, St Vincent's Hospital, The Catholic University of Korea, Suwon, South Korea; ${ }^{2}$ Department of Thoracic and Cardiovascular Surgery, St Vincent's Hospital, The Catholic University of Korea, Suwon, South Korea
Correspondence: Ki-Dong Yoo Department of Internal Medicine, St Vincent's Hospital, 93-6, Ji-dong, Paldal-ku, Suwon, Gyunggi-do,

16247, South Korea

$\mathrm{Tel}+82312497139$

Fax +82312477139

Email cardioyoo@gmail.com
Purpose: Extracorporeal membrane oxygenation (ECMO) is used to treat patients in critical condition with cardiogenic shock. However, few studies have examined the effect of old age in ECMO survival. This study analyzed the impact of age on ECMO survival of patients with cardiac failure, and analyzed predictive factors for survival according to age.

Materials and methods: We retrospectively reviewed the medical records of 95 patients who required veno-arterial (V-A) ECMO between May 2009 and May 2016 at a single center. Patients were classified into "age $\geq 65$ " ( $(\mathrm{n}=48,50.5 \%)$ and "age $<65$ " ( $\mathrm{n}=47,49.5 \%)$ groups.

Results: The age $\geq 65$ group was significantly associated with increased mortality (HR: 1.715; $95 \% \mathrm{CI}=1.038-2.831$ ) at 90 days after ECMO initiation. These associations were attenuated and did not retain statistical significance after adjustment for comorbidities (HR: 1.485; 95\% $\mathrm{CI}=0.844-2.614)$. To determine predictive factors of mortality, multivariate logistic analysis revealed that age $\geq 65$ (OR 5.750; 95\% CI [1.508-21.920]; $P=0.010$ ), low pre-ECMO serum bicarbonate (OR 0.884; 95\% CI [0.788-0.991]; $P=0.035$ ), and high pre-ECMO serum creatinine (OR 4.546; 95\% CI [1.021-20.239]; $P=0.047$ ) were significantly associated with survival to 90 days. By analyzing two groups separately, high pre-ECMO serum potassium level (OR 3.552; 95\% CI [1.023-12.331]; $P=0.046$ ) was the only independent predictor in patients aged $<65$ years while low Glasgow Coma Scale score (OR 0.698; 95\% CI [0.478-1.019]; $P=0.063$ ) showed a considerable trend toward significance in patients aged $\geq 65$.

Conclusion: Older age was not an independent risk factor for mortality at 90 days among V-A ECMO patients. In addition, our study provides understanding of the differences in predictive factors for ECMO survival according to age. Pre-ECMO laboratory findings and mental status can assist clinicians in the prediction of a patient's prognosis.

Keywords: extracorporeal membrane oxygenation, mortality, age

\section{Introduction}

Extracorporeal membrane oxygenation (ECMO) is used to treat patients in critical condition with life-threatening respiratory failure or cardiogenic shock. In particular, veno-arterial ( $\mathrm{V}-\mathrm{A}$ ) ECMO is increasingly being utilized to treat patients whose cardiopulmonary failure is refractory to conventional care, ${ }^{1,2}$ and improves the outcome of cardiac resuscitation in patients with cardiogenic shock or arrest due to ischemic heart disease..$^{3,4}$

While the acute survival benefit of V-A ECMO has been demonstrated, ${ }^{5}$ there have been few studies on elderly patients. ${ }^{6-9}$ Moreover, indications for the use of ECMO have yet to be established, and the use of V-A ECMO in elderly patients remains controversial. According to Extracorporeal Life Support Organization guidelines, advanced age is a contraindication for cardiac failure, whereas there are no restrictions on age for 
respiratory failure. ${ }^{10}$ However, the importance of ECMO for cardiopulmonary resuscitation is expected to rise due to increased life expectancies.

Previous studies have reported that V-A ECMO is also effective at resuscitating elderly patients, yielding a survival rate similar to that of younger patients. ${ }^{7,11}$ However, they did not present the differences of predictive factors for survival between older and younger patients. The purpose of this study was to investigate the impact of age on V-A ECMO survival of patients with cardiac failure. Furthermore, we analyzed the predictive factors for survival by dividing patients into two groups according to age.

\section{Materials and methods Study population}

This was a retrospective, single-center, observational study which was conducted between May 2009 and May 2016. Patients aged 18 years or older who underwent ECMO were enrolled. We excluded subjects with veno-venous ECMO $(\mathrm{n}=19)$. In total, $95 \mathrm{~V}-\mathrm{A}$ ECMO patients were included in the study. Data obtained from medical records, clinical case histories, and laboratory investigations were reviewed retrospectively. Follow-up data were collected from outpatient clinic records or via the telephone. The composite outcome was all-cause mortality at 90 days post-ECMO initiation. This study was approved by the Institutional Review Board of the Catholic University of Korea, St Vincent's Hospital (IRB approval number: VC16RISI0131). The study was exempted from requirements for written informed consent because we reviewed the medical records retrospectively. All data records were de-identified and analyzed anonymously.

\section{ECMO and management}

The ECMO team consisted of interventional cardiologists, cardiovascular surgeons, and perfusionists. Use of ECMO is usually considered under conditions of cardiac failure that is refractory to conventional care; typical causes are acute myocardial infarction (AMI), myocarditis, pulmonary thromboembolism (PTE), decompensated chronic heart failure, and post-cardiotomy shock. A Capiox emergency bypass system (Terumo, Tokyo, Japan) was used in all cases. Anticoagulation was accomplished through a bolus (100 units $/ \mathrm{kg}$ ) injection of unfractionated heparin followed by continuous intravenous infusion to maintain an activated clotting time of between 180 and $220 \mathrm{sec}$. Successful weaning was defined as separation from ECMO without reinsertion of ECMO or mortality within $24 \mathrm{hrs}$. All patients underwent blood transfusion to maintain a hematocrit level of $30 \%-35 \%$, and platelets were infused when the platelet count was $<100,000 / \mu \mathrm{L}$. Urine output, arterial blood gas analysis, electrolyte, and serum glucose levels were also monitored.

\section{Statistical analysis}

To investigate the baseline characteristic differences in age, continuous variables were expressed as the median and inter-quartile ranges or the mean $\pm \mathrm{SD}$, and compared using Mann-Whitney test or Student's $t$-test. Categorical variables were expressed as numbers and percentages, and compared using the chi-square test or Fisher's exact test. The survival of groups according to age was determined using the Kaplan-Meier method and compared using a log-rank test. HRs of a composite outcome were calculated with Cox regression models. We performed pre-specified analyses stratified according to the following variables: age ( $\geq 65$ years vs $<65$ years), sex, myocarditis or PTE, AMI, diabetes mellitus (DM), hypertension. Logistic regression analysis was performed to determine independent predictors associated with survival. Variables with significant associations using univariate analysis $(P<0.05)$ were entered into the multivariate analysis. ORs and $95 \%$ CIs were also calculated. A $P$-value $<0.05$ was considered statistically significant. Analyses were performed using Statistical Analysis Software (SAS, version 9.2, SAS Institute Inc., Cary, NC, USA).

\section{Results}

\section{Baseline patient characteristics}

Baseline patient characteristics are shown in Table 1. Patients were classified into "age $\geq 65$ " ( $\mathrm{n}=48,50.5 \%)$ and "age $<65$ " $(n=47,49.5 \%)$ groups. There was no difference in ECMO weaning between those two groups (age $<65,24 / 47,51.1 \%$ and age $\geq 65,16 / 48,33.3 \%, P=0.354$ ). However, there was a significant difference in the number of older patients (age $\geq 65$ years) who died during their hospital stay (age $<65$, $25 / 47,53.2 \%$ and age $\geq 65,40 / 48,83.3 \%, P=0.002)$. And more patients in the age $\geq 65$ group had DM and hypertension compared with the age $<65$ group.

\section{Survival outcomes}

Figure 1 shows the higher survival rate at 90 days in the age $<65$ group in V-A ECMO patients (log-rank, $P=0.022$ ). Univariate Cox regression analyses showed that age $\geq 65$ group was significantly associated with increased mortality (HR: 1.715; 95\% CI =1.038-2.831) (Table 2). However, after adjusting for comorbidities, the associations between age $\geq 65$ group and increased mortality were attenuated (HR: 1.485; 95\% CI =0.844-2.614) (Table 3). 
Table I Baseline characteristics

\begin{tabular}{|c|c|c|c|}
\hline Variables & $\begin{array}{l}\text { Age }<65 \\
(n=47)\end{array}$ & $\begin{array}{l}\text { Age } \geq 65 \\
(n=48)\end{array}$ & $P$-value \\
\hline Male, n (\%) & $31(66.0)$ & $28(58.3)$ & 0.444 \\
\hline Mean age (years), mean \pm SD & $49.2 \pm 10.9$ & $74.8 \pm 5.8$ & $<0.001$ \\
\hline ECMO indication & & & 0.008 \\
\hline Myocarditis, n (\%) & $8(17.0)$ & $0(0.0)$ & 0.003 \\
\hline PTE, n (\%) & $7(14.9)$ & $5(10.4)$ & 0.511 \\
\hline AMI, n (\%) & $18(38.3)$ & $31(64.6)$ & 0.010 \\
\hline Decompensated HF, n (\%) & $0(0.0)$ & $4(8.3)$ & 0.117 \\
\hline Post-cardiotomy, n (\%) & $3(6.4)$ & $2(4.2)$ & 0.677 \\
\hline Idiopathic VT/V.fib, n (\%) & $4(8.5)$ & $2(4.2)$ & 0.435 \\
\hline Other, n (\%) & $7(14.9)$ & $4(8.3)$ & 0.318 \\
\hline Current smoker, n (\%) & $27(57.4)$ & $20(4 \mid .7)$ & 0.124 \\
\hline CAD, n (\%) & $6(12.8)$ & $14(29.2)$ & 0.050 \\
\hline CVA, n (\%) & $\mathrm{I}(2.1)$ & $6(12.5)$ & 0.053 \\
\hline DM, n (\%) & $7(14.9)$ & $17(35.4)$ & 0.021 \\
\hline Hypertension, n (\%) & $14(29.8)$ & $32(66.7)$ & $<0.001$ \\
\hline CKD, n (\%) & $0(0.0)$ & $3(6.3)$ & 0.082 \\
\hline Cardiac arrest, n (\%) & $29(61.7)$ & $27(56.3)$ & 0.589 \\
\hline IHCA, n (\%) & $17(63.2)$ & $17(35.4)$ & \\
\hline OHCA, n (\%) & $12(25.5)$ & $10(20.8)$ & \\
\hline CA to ROSC time (min) (IQR) & $40.0(20.0-62.0)$ & $38.0(18.0-55.0)$ & 0.676 \\
\hline ECG before ECMO & & & 0.147 \\
\hline Asystole, n (\%) & $7(14.9)$ & $10(20.8)$ & \\
\hline PEA, n (\%) & $9(19.2)$ & $10(20.8)$ & \\
\hline VT/V.fib, n (\%) & $9(19.2)$ & $2(4.2)$ & \\
\hline Other, $\mathrm{n}(\%)$ & $22(46.8)$ & $26(54.2)$ & \\
\hline GCS score before ECMO, mean \pm SD & $8.0 \pm 5.1$ & $7.7 \pm 5.0$ & 0.826 \\
\hline APACHE II score, mean \pm SD & $18.9 \pm 7.2$ & $21.5 \pm 7.8$ & 0.093 \\
\hline \multicolumn{4}{|l|}{ Pre-ECMO laboratory finding } \\
\hline $\mathrm{pH}$, mean $\pm \mathrm{SD}$ & $7.2 \pm 0.2$ & $7.3 \pm 0.2$ & 0.227 \\
\hline $\mathrm{HCO} 3(\mathrm{mmol} / \mathrm{L})$, mean $\pm \mathrm{SD}$ & $14.4 \pm 5.0$ & $15.5 \pm 5.8$ & 0.365 \\
\hline Potassium (mEq/L), mean $\pm S D$ & $4.2 \pm 0.8$ & $4.3 \pm 1.0$ & 0.835 \\
\hline Creatinine $(\mathrm{mg} / \mathrm{dL})$, mean \pm SD & $1.3 \pm 0.7$ & $1.5 \pm 1.2$ & 0.499 \\
\hline Platelet $\left(\times 10^{9} / \mathrm{L}\right)(\mathrm{IQR})$ & $181.0(|| \mid 1.0-253.0)$ & $163.5(\mid 23.5-230.0)$ & 0.832 \\
\hline Total bilirubin (mg/dL) (IQR) & $0.6(0.4-1.2)$ & $0.8(0.5-1.1)$ & 0.793 \\
\hline CK-MB (ng/mL) (IQR) & $10.2(3.1-37.7)$ & $9.9(3.0-48.1)$ & 0.845 \\
\hline ECMO weaning, n (\%) & $24(5 \mathrm{I} .1)$ & $16(33.3)$ & 0.354 \\
\hline ECMO duration (days), mean \pm SD & $7.1 \pm 6.3$ & $8.1 \pm 7.5$ & 0.514 \\
\hline Death in hospital, n (\%) & $25(53.2)$ & $40(83.3)$ & 0.002 \\
\hline \multicolumn{4}{|l|}{ Complications } \\
\hline AKI, n (\%) & $21(44.7)$ & $20(4 \mid .7)$ & 0.767 \\
\hline CRRT, n (\%) & $17(36.2)$ & $14(29.2)$ & 0.467 \\
\hline Cannula site bleeding, n (\%) & II (23.4) & $8(16.7)$ & 0.412 \\
\hline
\end{tabular}

Abbreviations: ECMO, extracorporeal membrane oxygenation; PTE, pulmonary thromboembolism; AMI, acute myocardial infarction; HF, heart failure; VT, ventricular tachycardia; V.fib, ventricular fibrillation; CAD, coronary artery disease; CVA, cerebrovascular accident; DM, diabetes mellitus; CKD, chronic kidney disease; IHCA, in-hospital cardiac arrest; OHCA, out of hospital cardiac arrest; CA, cardiac arrest; ROSC, return of spontaneous circulation; IQR, inter-quartile range; ECG, electrocardiography; PEA, pulseless electrical activity; GCS, Glasgow Coma Scale; APACHE II, Acute Physiology and Chronic Health Evaluation II; CK-MB, creatine kinase MB; AKI, acute kidney injury; CRRT, continuous renal replacement therapy.

\section{Predictors of V-A ECMO mortality}

Independent predictors of V-A ECMO mortality were determined by univariate and multivariate logistic regression analyses. Age (especially $\geq 65$ ), history of hypertension, myocarditis or PTE for ECMO indication, low Glasgow Coma Scale (GCS) score, high Acute Physiology and Chronic Health Evaluation II (APACHE II) score, low pre-ECMO serum bicarbonate, and high pre-ECMO serum creatinine were significant univariate predictors of mortality (Table 4). Among these factors, multivariate analysis revealed that age $\geq 65$ (OR 5.750; 95\% CI [1.508-21.920]; $P=0.010$ ), low pre-ECMO serum bicarbonate (OR 0.884; 95\% CI [0.7880.991]; $P=0.035$ ), and high pre-ECMO serum creatinine (OR 4.546; 95\% CI [1.021-20.239]; $P=0.047$ ) were independent predictors of mortality of V-A ECMO (Table 4). To estimate the differences of predictors according to the group divided 


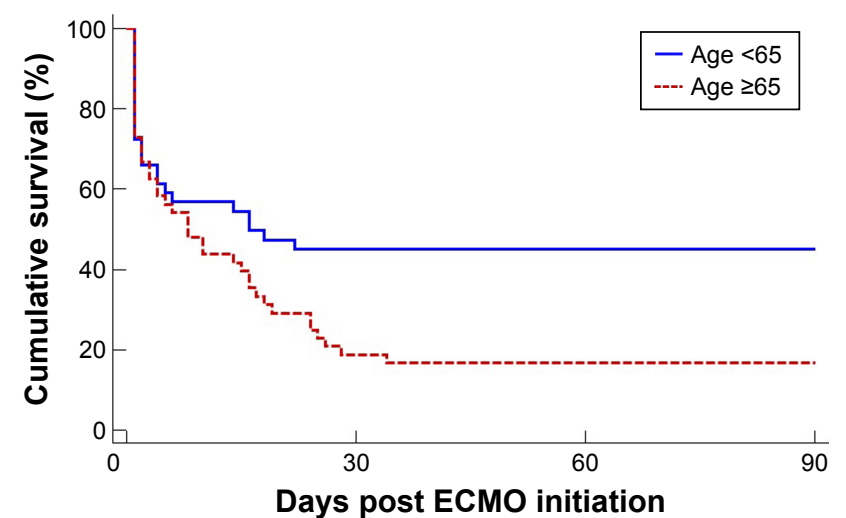

Number at risk

Group: age $<65$ 47

Group: age $\geq 65$ 48

17

9

17

Figure I Kaplan-Meier survival curves for 90-day mortality according to age. Note: Kaplan-Meier analysis showing the survival rate of patients aged $\geq 65$ years $(n=48)$ compared with those aged $<65$ years $(n=47)$.

Abbreviation: ECMO, extracorporeal membrane oxygenation.

by age $\geq 65$, multivariate logistic regression analysis was performed within each group. In the age $<65$ group, bicarbonate, potassium, creatinine level, and APACHE II score were significant univariate predictors of mortality (Table 5). However, high pre-ECMO serum potassium level (OR 3.552; 95\% CI [1.023-12.331]; $P=0.046$ ) was the only independent predictor of mortality of V-A ECMO in the age $<65$ group, while low GCS score (OR 0.698; 95\% CI [0.478-1.019]; $P=0.063$ ) showed only a considerable trend toward significance in the age $\geq 65$ group (Table 6 ).

\section{Discussion}

In our study, we investigated the impact of age on V-A ECMO survival of patients with cardiac failure. We also analyzed the predictive factors of survival by dividing patients into two groups according to age. The age $\geq 65$ group showed a higher mortality rate; however, after adjusting for comorbidities, age was no longer an independent risk factor of mortality. Additionally, age $\geq 65$, low pre-ECMO serum bicarbonate, and high pre-ECMO serum creatinine were identified as independent predictors of mortality of V-A ECMO. Our study showed that the predictive factors of mortality differ between those two groups.

Table 2 Univariate Cox regression model

\begin{tabular}{llll}
\hline Age & HR & $95 \% \mathrm{Cl}$ & $P$-value \\
\hline$\geq 65$ & 1.715 & $1.038-2.831$ & 0.035 \\
\hline
\end{tabular}

Abbreviations: $\mathrm{HR}$, hazard ratio; $\mathrm{Cl}$, confidence interval.
Table 3 Multivariate Cox regression model

\begin{tabular}{llll}
\hline Variables & HR & $\mathbf{9 5 \%} \mathbf{C l}$ & $P$-value \\
\hline Age $\geq 65$ & 1.485 & $0.844-2.614$ & 0.170 \\
Sex & 1.029 & $0.593-1.786$ & 0.919 \\
Myocarditis or PTE & 0.548 & $0.230-1.305$ & 0.174 \\
AMI & 1.074 & $0.611-1.886$ & 0.805 \\
DM & 0.855 & $0.480-1.522$ & 0.594 \\
Hypertension & 1.163 & $0.660-2.050$ & 0.602 \\
\hline
\end{tabular}

Abbreviations: PTE, pulmonary thromboembolism; AMI, acute myocardial infarction; DM, diabetes mellitus; HR, hazard ratio.

V-A ECMO has been successfully used to treat cardiogenic shock and cardiac arrest refractory to usual resuscitation, and the survival benefit of V-A ECMO has been demonstrated. ${ }^{5}$ However, there are few studies on elderly patients. ${ }^{6-9}$ Moreover, indications for the use of ECMO are still unclear, and the use of V-A ECMO for elderly patients remains controversial. However, the importance of ECMO for cardiopulmonary resuscitation is expected to rise as society ages.

Old age is a well-known predictive factor for poor survival of ECMO. ${ }^{12}$ In addition, some factors such as the APACHE score, acute kidney injury or renal replacement therapy, the existence of peripheral artery disease, and asystole or pulseless electrical activity before ECMO introduction, are associated with a worse prognosis. ${ }^{13-18}$ In contrast, acute myocarditis, high GCS score, and successful reperfusion therapy for acute coronary syndrome are associated with better prognosis. . $^{12,17,18}$

However, there are currently two studies that have reported that V-A ECMO in elderly patients is also effective, yielding a survival rate similar to that of younger patients. ${ }^{711}$ Saito et $\mathrm{al}^{7}$ conducted a single-center trial to evaluate the impact of V-A ECMO on the survival of patients with cardiogenic shock. They concluded that ECMO is also effective at resuscitating elderly patients, yielding a hospital survival rate similar to that of younger patients. However, in that study, the number of patients in the elderly group was relatively low, because the cut-off level for the elderly group was set at 75 years. Narotsky et al reported that old age was not independently associated with short-term or long-term survival among V-A ECMO patients. However, in that study, the laboratory findings, electrocardiogram, and underlying diseases before ECMO insertion were not recorded. In addition, specific multivariate models stratified by age group were not included. ${ }^{11}$ In our study, there was no significant difference in ECMO weaning between groups. However, more patients in the age $\geq 65$ group died during 
Table 4 Logistic regression analysis for independent predictors of V-A ECMO mortality in total

\begin{tabular}{|c|c|c|c|c|c|c|}
\hline \multirow[t]{2}{*}{ Variables } & \multicolumn{3}{|c|}{ Univariate } & \multicolumn{3}{|c|}{ Multivariate } \\
\hline & OR & $95 \% \mathrm{Cl}$ & $P$-value & OR & $95 \% \mathrm{Cl}$ & $P$-value \\
\hline Age $\geq 65$ & 4.400 & $1.700-11.389$ & 0.002 & 5.750 & $1.508-21.920$ & 0.010 \\
\hline Myocarditis or PTE & 0.278 & $0.100-0.772$ & 0.014 & 0.299 & $0.072-1.246$ & 0.097 \\
\hline Hypertension & 2.483 & $1.006-6.125$ & 0.048 & 1.831 & $0.503-6.667$ & 0.359 \\
\hline $\mathrm{HCO} 3$ & 0.909 & $0.835-0.990$ & 0.029 & 0.884 & $0.788-0.991$ & 0.035 \\
\hline Potassium & 1.713 & $0.97 I-3.023$ & 0.063 & & & \\
\hline Creatinine & 3.523 & $1.192-10.415$ & 0.023 & 4.546 & $1.021-20.239$ & 0.047 \\
\hline GCS score & 0.888 & $0.8|2-0.97|$ & 0.009 & 0.358 & $0.059-2.164$ & 0.263 \\
\hline Asystole or PEA & 2.647 & $0.996-7.033$ & 0.051 & & & \\
\hline APACHE II & 1.123 & $1.049-1.202$ & 0.001 & 1.020 & $0.896-1.162$ & 0.763 \\
\hline
\end{tabular}

Abbreviations: V-A ECMO, venous-arterial extracorporeal membrane oxygenation; PTE, pulmonary thromboembolism; GCS, Glasgow Coma Scale; PEA, pulseless electrical activity; APACHE II, Acute Physiology and Chronic Health Evaluation II.

their hospital stay. After adjusting for comorbidities, older age was not an independent risk factor of mortality at 90 days among V-A ECMO patients. These results were probably due to comorbidities with advanced age.

In the present study, age $\geq 65$, low pre-ECMO serum bicarbonate, and high pre-ECMO serum creatinine were identified as independent predictors of mortality of VA-ECMO. Moreover, our study shows that the predictive factors for mortality differ between groups. In the younger group, bicarbonate, potassium, creatinine level, and APACHE II score were significant univariate predictors of mortality. However, in the younger group, high pre-ECMO serum potassium level was the only independent predictor of V-A ECMO mortality, while low GCS score showed only a considerable trend toward significance in the elderly group. The clinical indices of metabolic acidosis such as lower serum bicarbonate, high serum anion gap or acidic urine $\mathrm{pH}$ have been associated with well-known risk factors for all-cause and cardiovascular mortality in a healthy population. ${ }^{19,20}$ These results may explain why low pre-ECMO serum bicarbonate was an independent predictor of mortality of VA-ECMO. In addition, in previous studies, it was shown that the anaerobic function of central nervous system white matter declines with age and favorable outcome after stroke and brain injuries declines with age. ${ }^{21-23}$ These results may explain why low GCS score was the important factor in mortality of older patients compared to younger patients. Meanwhile, serum potassium levels strongly influence the excitability of cardiomyocytes and muscle cells. Hyperkalemia in cardiogenic shock is related to acute kidney injury, breakdown of muscle fibers, and destruction of red blood cells. Moreover, hyperkalemia is associated with cardiovascular events and mortality. ${ }^{24}$ Usually, younger patients have more muscle mass than older patients. These results may explain why high potassium level was the important factor in mortality of younger patients.

The strength of the present study is that the laboratory findings and mental status before ECMO introduction were measured and investigated among a relatively large sample of elderly patients. However, the present study has several limitations. First, it is a retrospective observational study. However, ECMO support is a difficult problem with regard to randomization because the necessity for V-A ECMO often arises suddenly. The generalizability of results is increased by its observational status, representing real-world practice.

Table 5 Logistic regression analysis for independent predictors of V-A ECMO mortality in age $<65$ group

\begin{tabular}{|c|c|c|c|c|c|c|}
\hline \multirow[t]{2}{*}{ Variables } & \multicolumn{3}{|c|}{ Univariate } & \multicolumn{3}{|c|}{ Multivariate } \\
\hline & $\overline{\text { OR }}$ & $95 \% \mathrm{Cl}$ & $P$-value & $\overline{\text { OR }}$ & $95 \% \mathrm{Cl}$ & $P$-value \\
\hline Myocarditis or PTE & 0.300 & $0.083-1.090$ & 0.067 & & & \\
\hline $\mathrm{HCO} 3$ & 0.853 & $0.730-0.996$ & 0.045 & 0.861 & $0.707-1.050$ & 0.139 \\
\hline Potassium & 4.013 & $1.359-11.847$ & 0.012 & 3.552 & $|.023-| 2.33 \mid$ & 0.046 \\
\hline Creatinine & 5.517 & $1.148-26.513$ & 0.033 & 2.660 & $0.519-13.634$ & $0.24 I$ \\
\hline GCS & 0.925 & $0.824-1.039$ & 0.188 & & & \\
\hline Asystole or PEA & 2.671 & $0.749-9.529$ & 0.130 & & & \\
\hline APACHE ॥ & 1.118 & $1.018-1.227$ & 0.019 & 1.110 & $0.987-1.249$ & 0.083 \\
\hline
\end{tabular}

Abbreviations: V-A ECMO, venous-arterial extracorporeal membrane oxygenation; PTE, pulmonary thromboembolism; GCS, Glasgow Coma Scale; PEA, pulseless electrical activity; APACHE II, Acute Physiology and Chronic Health Evaluation II. 
Table 6 Logistic regression analysis for independent predictors of V-A ECMO mortality in age $\geq 65$ group

\begin{tabular}{|c|c|c|c|c|c|c|}
\hline \multirow[t]{2}{*}{ Variables } & \multicolumn{3}{|c|}{ Univariate } & \multicolumn{3}{|c|}{ Multivariate } \\
\hline & OR & $95 \% \mathrm{CI}$ & $P$-value & OR & $95 \% \mathrm{Cl}$ & $P$-value \\
\hline Cardiac arrest & 2.500 & $0.523-11.956$ & 0.251 & & & \\
\hline $\mathrm{HCO} 3$ & 0.893 & $0.782-1.021$ & 0.097 & & & \\
\hline Creatinine & 2.645 & $0.451-15.520$ & 0.281 & & & \\
\hline GCS & 0.787 & $0.652-0.950$ & 0.013 & 0.698 & $0.478-1.019$ & 0.063 \\
\hline Asystole or PEA & 2.455 & $0.44 I-I 3.670$ & 0.305 & & & \\
\hline APACHE II & 1.127 & $1.001-1.268$ & 0.049 & 0.916 & $0.728-1.152$ & 0.453 \\
\hline
\end{tabular}

Abbreviations: V-A ECMO, venous-arterial extracorporeal membrane oxygenation; GCS, Glasgow Coma Scale; PEA, pulseless electrical activity; APACHE II, Acute Physiology and Chronic Health Evaluation II.

Second, we did not calculate other scores such as the Simplified Acute Physiology Score II or Sepsis-related Organ Failure Assessment, which are known predictor factors of survival of ECMO. Third, the number of patients alive at 90 days is too small. The associations between age and increased mortality were attenuated in multivariate Cox regression analysis. This result was probably due to the small sample size and comorbidities with advanced age. We need large sample sizes to address this important issue. Finally, our study included heterogeneous patients such as both post-operative and non-operative, as well as both in-hospital cardiac arrest and out-of-hospital cardiac arrest. Despite these limitations, the present results emphasize the importance of understanding the impact of age in V-A ECMO survival in resuscitating patients and the differences among predictive factors of V-A ECMO mortality by dividing patients into two groups according to age.

In conclusion, older age was not an independent risk factor of mortality at 90 days among V-A ECMO patients after adjustment for comorbidities. In addition, our study provides understanding of the differences in predictive factors for ECMO survival according to age. Pre-ECMO laboratory findings and mental status can assist clinicians in the prediction of a patient's prognosis.

\section{Acknowledgments}

We acknowledge the helpful support of all authors.

\section{Disclosure}

The authors report no conflicts of interest in this work.

\section{References}

1. Mendiratta P, Tang X, Collins RT 2nd, Rycus P, Brogan TV, Prodhan P. Extracorporeal membrane oxygenation for respiratory failure in the elderly: a review of the Extracorporeal Life Support Organization registry. ASAIO J. 2014;60(4):385-390.

2. Sauer CM, Yuh DD, Bonde P. Extracorporeal membrane oxygenation use has increased by $433 \%$ in adults in the United States from 2006 to 2011. ASAIO J. 2015;61(1):31-36.
3. Rees MR, Browne T, Sivananthan UM, et al. Cardiac resuscitation with percutaneous cardiopulmonary support. Lancet. 1992;340(8818): 513-514.

4. Shawl FA, Domanski MJ, Hernandez TJ, Punja S. Emergency percutaneous cardiopulmonary bypass support in cardiogenic shock from acute myocardial infarction. Am J Cardiol. 1989;64(16): 967-970.

5. Cheng R, Hachamovitch R, Kittleson M, et al. Complications of extracorporeal membrane oxygenation for treatment of cardiogenic shock and cardiac arrest: a meta-analysis of 1,866 adult patients. Ann Thorac Surg. 2014;97(2):610-616.

6. Beurtheret S, Mordant P, Paoletti X, et al. Emergency circulatory support in refractory cardiogenic shock patients in remote institutions: a pilot study (the cardiac-RESCUE program). Eur Heart J. 2013;34(2): 112-120.

7. Saito S, Nakatani T, Kobayashi J, et al. Is extracorporeal life support contraindicated in elderly patients? Ann Thorac Surg. 2007;83(1): $140-145$.

8. Stohr F, Emmert MY, Lachat ML, et al. Extracorporeal membrane oxygenation for acute respiratory distress syndrome: is the configuration mode an important predictor for the outcome? Interact Cardiovasc Thorac Surg. 2011;12(5):676-680.

9. Shin TG, Choi JH, Jo IJ, et al. Extracorporeal cardiopulmonary resuscitation in patients with inhospital cardiac arrest: A comparison with conventional cardiopulmonary resuscitation. Crit Care Med. 2011;39(1): $1-7$.

10. Extracorporeal Life Support Organization [homepage on the Internet]. ELSO Guidelines. ELSO; 2015. Available from: https://www.elso.org/ resources/guidelines.aspx. Accessed July 6, 2017.

11. Narotsky DL, Mosca MS, Mochari-Greenberger H, et al. Short-term and longer-term survival after veno-arterial extracorporeal membrane oxygenation in an adult patient population: does older age matter? Perfusion. 2016;31(5):366-375.

12. Chen YC, Tsai FC, Chang CH, et al. Prognosis of patients on extracorporeal membrane oxygenation: the impact of acute kidney injury on mortality. Ann Thorac Surg. 2011;91(1):137-142.

13. Bisdas T, Beutel G, Warnecke G, et al. Vascular complications in patients undergoing femoral cannulation for extracorporeal membrane oxygenation support. Ann Thorac Surg. 2011;92(2):626-631.

14. Chang WW, Tsai FC, Tsai TY, et al. Predictors of mortality in patients successfully weaned from extracorporeal membrane oxygenation. PLoS One. 2012;7(8):e42687.

15. Chung SY, Sheu JJ, Lin YJ, et al. Outcome of patients with profound cardiogenic shock after cardiopulmonary resuscitation and prompt extracorporeal membrane oxygenation support. A single-center observational study. Circ J. 2012;76(6):1385-1392.

16. Lin CY, Tsai FC, Tian YC, et al. Evaluation of outcome scoring systems for patients on extracorporeal membrane oxygenation. Ann Thorac Surg. 2007;84(4):1256-1262.

17. Sakamoto S, Taniguchi N, Nakajima S, Takahashi A. Extracorporeal life support for cardiogenic shock or cardiac arrest due to acute coronary syndrome. Ann Thorac Surg. 2012;94(1):1-7. 
18. Thiagarajan RR, Brogan TV, Scheurer MA, Laussen PC, Rycus PT, Bratton SL. Extracorporeal membrane oxygenation to support cardiopulmonary resuscitation in adults. Ann Thorac Surg. 2009;87(3): 778-785.

19. Raphael KL, Zhang Y, Wei G, Greene T, Cheung AK, Beddhu S. Serum bicarbonate and mortality in adults in NHANES III. Nephrol Dial Transplant. 2013;28(5):1207-1213.

20. Park M, Jung SJ, Yoon S, Yun JM, Yoon HJ. Association between the markers of metabolic acid load and higher all-cause and cardiovascular mortality in a general population with preserved renal function. Hypertens Res. 2015;38(6):433-438.

21. Hamner MA, Moller T, Ransom BR. Anaerobic function of CNS white matter declines with age. J Cereb Blood Flow Metab. 2011;31(4): 996-1002.
22. Sendroy-Terrill M, Whiteneck GG, Brooks CA. Aging with traumatic brain injury: cross-sectional follow-up of people receiving inpatient rehabilitation over more than 3 decades. Arch Phys Med Rehabil. 2010; 91(3):489-497.

23. Dhamoon MS, Moon YP, Paik MC, et al. Long-term functional recovery after first ischemic stroke: the Northern Manhattan Study. Stroke. 2009;40(8):2805-2811.

24. Hughes-Austin JM, Rifkin DE, Beben T, et al. The relation of serum potassium concentration with cardiovascular events and mortality in community-living individuals. Clin J Am Soc Nephrol. 2017;12(2): $245-252$.
Clinical Interventions in Aging

\section{Publish your work in this journal}

Clinical Interventions in Aging is an international, peer-reviewed journal focusing on evidence-based reports on the value or lack thereof of treatments intended to prevent or delay the onset of maladaptive correlates of aging in human beings. This journal is indexed on PubMed Central, MedLine,

\section{Dovepress}

CAS, Scopus and the Elsevier Bibliographic databases. The manuscript management system is completely online and includes a very quick and fair peer-review system, which is all easy to use. Visit http://www.dovepress. $\mathrm{com} /$ testimonials.php to read real quotes from published authors. 